KN Kartographische Nachrichten Journal of Cartography and Geographic Information 65. Jahrgang, Februar 2015, Heft 1 Herausgeber: Deutsche Gesellschaft für Kartographie e.V. (DGfK) - Gesellschaft für Kartographie und Geomatik vertreten für Kartographie und Geom
durch den Präsidenten Prof. Dr. Manfred Weisensee Prosellschaft für Kartographie eV (DGfK). Organ der Deutschen Gesellschaft für Kartographie eV. (DGIK), der Schweizerischen Gesellschaft für Kartografie (SGK) und der Osterreichischen Kartographischen Kommission (OKK)
Osterreichischen Gesellschaft für Geographie (OGG).

Osterreichisch

Schriftleitung

Prof Dr. rer nat, habil. Mark Vetter (zuständig für Aufsätze,

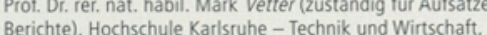
Berichte). Hochschule Karlsruhe - Technik und Wirtschaft,
Moltkestr. 30, 76133 Karlsruhe; redaktion@kartographischeMoltkestr. 30, 76133 Karlsruhe; redaktion
nachrichten.de, fon: $0049(0) 7219252599$

Weitere Schriftleiter

Prof. Dr. phil. Frank Dickmann (Zweiter Schriftleiter. Prof. Dr. phit. Frank Dickmann (Zweiter Schriftteiter, zuständig für Hochschulnachrichten, Rezensione: Ruhr-Universität Bochum, Geographisches Institut, Postfach, 44780 Bochum; frank.dickmann @irub.de Fon: 0049(0)234 32-23379; Fax: 0049(0)234 32-14964 Dipl.-Ing. Andreas Gollenstede (zuständig für Geoinformation aktuell) Haareneschstr. 91, 26121 Oldenburg: kn@gollenstede.com Fon: 0049(0)441 7779-545; Fax: 0049(0)441 7779-750 Dr. Thomas Chudy (zustāndig für DGfK-, SKG- und OGG. Nachrichten). Martin-Luther-Universităt Halle-Wittenberg, Institut für Agrar- und Ernährungswissenschaften, Karl-Freiherr-vonFritsch-Straße 4, 06120 Halle/ Saale, Tel:0049(0)345 5522448: Fax: $0049(0) 3455527109$

Mitarbeit für die Schweiz: Prof. Dr. Lorenz Hurni.

Institut für Kartografie, ETH Hönggerberg, 8093 Zürich; hurni@karto.baug.ethz.ch

Fon: 00411 6333033; Fax: 00411631153

Mitarbeit für Osterreich: Univ.-Prof. Dr. Wolfgang Kainz. Institut für Geographie und Regionalforschung. Universităt Wien, Universitătsstraße 7/5, 1010 Wien; wolfgang.kainz@univie.ac.at

Fon: 00431 4277-8640; Fax: 00431 4277-9531

Ehrenschriftleiter

Prof. i.R. Dr. Jürgen Dodt, Witten

Dr. rer. nat. h. c. Rolf Harbeck, Bonn

Editorial Board

Prof. Dr. H. Asche, Potsdam; Prof. Dr. Manfred Buchroithner, Dresden; Prof i. R. Dr. Jürgen Dodt, Bonn; Prof. Dr. D. Dransch, Dresden;

Prof. Dr. M. Ehlers, Osnabrück, Berlin; Prof. Dr. S. I. Fabrikant, Zürich,

Schweiz; Ass.-Prof. Dr. S. Fuhrmann, Fairfax, USA; Prof. Dr. G. Gartner,

Wien, Osterreich; Prof. Dr. D. Grünreich, Ronnenberg; Dr. Rolf Harbeck,

Bonn; Prof. Dr. A. Hüttermann, Ludwigsburg: Prof. Dr. L. Hurni, Zürich;

Prof. Dr. W. Kainz, Wien; Prof. Dr. P. Kammerer, München;

Prof. Dr. W. G. Koch, Dresden; Prof. Dr.-Ing. L. Meng, München;

Prof. Dr. J. Schiewe, Hamburg; PD Dr.-Ing. J. Schoppmeyer, Bonn:

Prof. Dr. J Schweikart Berlin:Prof. Dr.-Ing. M. Sester. Hannover:

Prof. Dr. J. Siemer, Regina, Kanada; Prof. Dr. R. Zölitz, Greifswald.

Manuskripte richten Sie bitte an den zuständigen Schriftleiter

Hinweise zur Gestaltung des Manuskripts finden Sie unter

mww.kartographische-nachrichten de $>$ Autorenhinweise.

Reviewverfahren

Wissenschaftliche Beitrăge werden im Rahmen eines Peer-ReviewVerfahrens begutachtet. Trotz sorgfaltiger Redigierung übernehmen Herausgeber, Redaktion und Verlag keine Haftung für die inhaltlich Richtigkeit der Veröffentlichungen.

Die KN Kartographische Nachrichten ist in der internationalen Zitationsdatenbank Scopus gelistet.

Die DGfK ist Mitglied der

Internationalen Kartographischen

Vereinigung IKV/ICA.

Verlag: Kirschbaum Verlag GmbH, Siegfried.

straße 28, D-53179 Bonn / Postfach 210209, D-53157 Bonn,

Tel.: (0228) 95453-0, Fax: (0228)95453-27:

Internet: http://www.kirschbaum.de, E-Mail: info@kirschbaum.de

Anzeigenleiter: Volker Rutkowski; zurzeit ist Anzeigenpreisliste

Nr. 50 vom 1.10.2014 gültig

Bezugspreise und ISSN: Inland 67,70 Euro jährlich, inkl. MwSt. und Versandkosten; Einzelheft 12,- Euro inkl. MwSt.

Ausland 69,80 Euro jährlich, inkl. MwSt. und Versandkosten:

Einzelheft 12,- Euro inkl. MwSt.

Die Zeitschrift erscheint alle zwei Monate.

ISSN 0022-9164.

Satz: Mohr Mediendesign, Siebengebirgsweg 40, D-53773 Hennef, Internet: www.mohrmediendesign.de

Druck: SDV Saarländische Druckerei und Verlag GmbH,

Werner-von-Siemens-StraBe 31, D-66793 Saarwellingen

\section{KN - Der Wechsel der Schriftleitung}

\section{Liebe Leserin, lieber Leser,}

Sie haben es sicher gleich bemerkt: Neben diesem Text steht nicht mehr das Ihnen vertraute Bild von Dipl.-Ing. Dr. h.c. Rolf Harbeck, sondern meines. Als neuer Schriftleiter der $\mathrm{KN}$ möchte ich mich Ihnen kurz vorstellen:

1991 habe ich als Geographiestudent im 1. Semester an der Humboldt-Universität zu Berlin meine erste Vorlesung in Kartographie gehört. Schnell hat mich die Begeisterung erfasst, räumliche Phänomene im Kartenbild exakt oder thematisch relevant einzuordnen oder darzustellen.

Schon während meines Studiums verspürte ich das Interesse, wissenschaftlich zu arbeiten. So folgte ich 1998 Prof. Dr. Otfried Baume, dem Betreuer meiner Diplomarbeit und späteren Doktorvater, an die LMU nach München, um zu einem Forschungsprojekt über den Königssee im Nationalpark Berchtesgaden methodisch unter Berücksichtigung von GIS-Techniken zu arbeiten. Bereits im Rahmen meines Promotionsprojektes, welches ich 2003 abschließen konnte, habe ich mich mit dem Geoprocessing bzw. der räumlichen Analyse im GIS, gewissermaßen also mit der elektronischen Kartographie, beschäftigt.

Nach verschiedenen Gastprofessuren an Hochschulen im In- und Ausland konnte ich 2010 meine Habilitation im

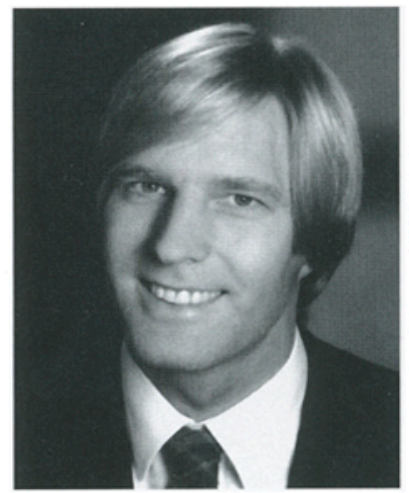
Fach Geographie an der LMU abschließen. Seit März 2013 bin ich als Professor an der Hochschule für Angewandte Wissenschaften Karlsruhe - Technik und Wirtschaft unter der Denomination ,Kartographie und Geovisualisierung' tätig. Schwerpunkte in der Lehre sind Vorlesungen in der Kartographie und GIS-Praktika.

Als Hauptschriftleiter ist mir sehr schnell bewusst geworden, dass ich diese Aufgabe nicht alleine erfolgreich bewältigen kann, sondern auf vielfältige Unterstützung angewiesen bin. Die Vorstandskollegen der DGfK unterstützen mich mit vereinten Kräften bei diesem Bemühen, ebenso steht mir glücklicherweise eine Redaktionsassistenz zur Seite. Allein diese Hilfe würde aber nicht ausreichen. Daher haben wir in der Redaktion zusätzlich noch ein Editorial Management System (EMS) und eine professionelle Lektorats-Software eingeführt. Mein Dank für die Unterstützung gilt an dieser Stelle meinen Redaktionskollegen Prof. Dr. Frank Dickmann, Dipl.-Ing. Andreas Gollenstede und Dr. Thomas Chudy für die Erstellung von Beiträgen für die KN. Nicht alle können hier genannt werden, denen ich meinen Dank schulde, den komplexen Organismus zur Erstellung einer Zeitschrift am Laufen zu halten. Ich wäre zu Beginn meiner Tätigkeit hoffnungslos überfordert gewesen, wenn nicht Rolf Harbeck mich so geduldig und umfassend in die Redaktionsarbeit eingeführt hätte. Ihm ist es zu verdanken, dass das Haus KN so gut bestellt ist. Einen persönlichen, sehr herzlichen Dank, lieber Rolf, für Dein Engagement!

Es ist mir eine große Ehre, dass mich der Präsident der DGfK, Prof. Dr.-Ing. Manfred Weisensee gefragt hat, ob ich mir vorstellen könnte die Schriftleitung der $\mathrm{KN}$ zu übernehmen. Sehr gerne nehme ich diese Herausforderung an und freue mich auf viele interessante Hefte!

Einige Worte zum vorliegenden Heft: Gleich in zwei Artikeln wird die Thematik VGI (Volunteered Geographic Information) aus recht unterschiedlichen Perspektiven behandelt. Während Markus Mayr die lizenzrechtlich schwierige Situation bei diesen Geoinformationen beleuchtet, stellt das Autorenteam der Kollegen Matthias Möller und Sven Fuhrmann die gewinnbringende Verwendungsmöglichkeit dieser Daten in Krisensituationen dar. Ein weiterer Aufsatz vom Autorenkonsortium um Matthias Möller behandelt Möglichkeiten, den historischen, häufig analogen Kartenschatz für die Gegenwart und Zukunft auf vielfältige Weise auch in digitaler Form nutzbar zu machen. Schließlich stellt der Beitrag von Stefan Peters dar, welche neuen visuell-analytischen Lösungen zur Untersuchung von Blitz-Trajektorien angewandt werden können.

Ich wünsche Ihnen viel Freude beim Lesen des vorliegenden Heftes!

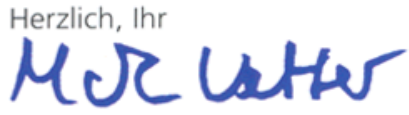

